\title{
Electricity Is Not What You Think
}

\author{
Michael Bank \\ Jerusalem College of Technology, Jerusalem, Israel \\ Email: bankmichael1@gmail.com
}

How to cite this paper: Bank, M. (2019) Electricity Is Not What You Think. Engineering, 11, 801-805.

https://doi.org/10.4236/eng.2019.1112054

Received: October 31, 2019

Accepted: November 23, 2019

Published: November 26, 2019

Copyright $\odot 2019$ by author(s) and Scientific Research Publishing Inc. This work is licensed under the Creative Commons Attribution International License (CC BY 4.0).

http://creativecommons.org/licenses/by/4.0/

\begin{abstract}
This article is an attempt to answer student's (and not only students') questions about the possibility of inventions nowadays in the field of electricity. The student is confident that nowadays everything is clear. Large firms do everything which is needed. And the time for inventions is over. But in fact, many things are not so clear, and a new Tesla may appear.
\end{abstract}

\section{Keywords}

Grounding, One Wire, SWER, Three Phase, Wide Band Antenna

\section{Introduction}

It is written in the books that electrical energy is transmitted through the wires, but what exactly does flow inside the wire?

Today there exist generally accepted descriptions of processes occurring in an electric circuit. These descriptions are based on a model in which electrons (or other charges) move inside a conductor. In some literature it is written that the electrons do not move, but push each other, as in the well-known "domino effect".

But such an explanation is not plausible. Electrons are mechanical particles that have mass. They cannot move or push each other at the speed of light. But an electrical signal is transmitted at the speed of light.

If so, then how electrical energy is transmitted in a wire system?

Here is a modern explanation of how electrical energy is transmitted in wires. The source creates a potential difference. The potential difference creates an electromagnetic field. This field propagates along the wire on its outer surface at the speed of light (this creates a well-known skin effect). So, wire is not a tube with a current inside. The current does not exist at all [1].

\section{Does One Need Grounding?}

There is a common opinion that all electrical systems and all devices must have 
connection with ground. But at the same time, there are contradictions in the explanations of grounding systems functioning. In these systems, the current enters the earth. But today it is known that it is impossible to find out any traces of this current at distance of a few meters. If so, where does the current go? There are many attempts to explain these processes. And all these explanations are different. Some people assume that the earth is a huge capacitor. But, firstly a capacitor should have the second plate. And, secondly, there should be a dielectric inside a capacitor. But the earth is not a dielectric. Other explanations assume that grounding as current absorption. But absorption cannot be infinite. Any sponge, when it is filled with water, stops absorbing. There are other explanations as well, but all of them cause new questions. Let's consider the following hypothesis [2].

The current which is injected into a ground is divided into a great number of weak currents. When ground depth increases, the quantity of currents grows and therefore the amplitude of each current decreases to zero. Can current flow through broken wires? Yes, it can, for example, in a linear antenna. In the case of electrical antenna like a dipole or monopole, the current stops at the ends of radiators but its energy converts into the energy of the electromagnetic field. It means that the energy path is not interrupted.

Now we can imagine a grounding or zeroing as a set of very short monopoles. It is known that monopole which height $(\mathrm{h})$ is much less than a quarter of wavelength has radiation resistance close to zero. Recall that the wavelength at a frequency of $50 \mathrm{~Hz}$ is 6000 kilometers. This resistance tends to zero as $\mathrm{h}$ (compared to the quarter of wavelength) decreases. Radiation resistance decrease leads to radiating power decrease. So, we can say that monopole with $5-10 \mathrm{~m}$ high at frequencies 50 or $60 \mathrm{~Hz}$ has zero resistance and zero radiation field density. Note that a monopole with height much less than the quarter of wavelength has a capacitive component. However, parallel connection of monopoles results in capacitive resistance decrease.

In other words, perhaps grounding is a system consisting of a considerable quantity of monopoles, with length much smaller than quarter of a wavelength.

\section{An Unbalanced Single Wire Earth Return (SWER) System}

In SWER system, the energy is transmitted by one wire. The second source terminal and the second load terminal are connected to the ground. This unbalanced line can transmit all the energy from source Tx to load $\mathrm{Rx}$. But there is one problem. Potentials of grounding in the $\mathrm{Tx}$ and $\mathrm{Rx}$ are the same (zero). However, potentials in active terminals of transmitter and in receiver are different due to a signal delay. So, $\mathrm{Tx}$ and $\mathrm{Rx}$ signals have a different potential difference. It shall be noted, that in the balanced circuit with active load, the potential difference on receiver input does not depend on signal delay, as potentials at the ends of both wires change equally by a delay. This situation can be likened to the problems similar to reactive load influence. In the case of a reactive load, there is 
a reactive energy, namely, the source must produce active and reactive energy. And in the case of long lines, this system loses a large amount of energy. It is possible to compensate reactive power, but this compensation is expensive in the case of variable loads. In contrast to the SWER system, the proposed one-wire system [3] is a balanced system. In it, the potential difference on the load does not depend on the line length and is always equal to 180 degrees.

\section{Three Phase System: Is It Good or Not Very Good?}

Now the dominant electric energy transmission system is the three-phase system proposed by the Russian scientist Dolivo-Dobrovolsky. The important advantage of this system is that a generator and an electric motor are effectively built, because in these devices, three windings are located on one rotor. But today it is already clear to many specialists, that this system has more disadvantages than advantages. Here are these disadvantages:

- Many expensive wires (three or four);

- Large expensive supports for wires;

- Underground and underwater three phase system are very expensive;

- Large number of broken wires short circuits;

- Large energy losses due to phase's imbalances and reactive power;

- Strong negative environmental impact including Corona Effect, it's time to free the earth (see Figure 1).

In this case, one can say that three phase system is three SWER systems.

As in SWER, this phenomenon is equivalent to the presence of reactive resistance in the load and, consequently, the appearance of reactive power. It is shown in [1] that reactive power value in three phase system noticeably increases if cables have linear capacitances and linear inductances. That is why there are intermediate stations sometimes every about $30 \mathrm{~km}$.

One-wire system allows transmitting the energy from three phase source by one wire. In this case in one wire one can use the one of three wires of equivalent three phase system [1]. For transmitting three phase signals by one wire signal it is possible using special converter [4].

\section{One Wire System}

In [5] universal balanced one wire system is proposed. All loads receive signals with phase difference 180 or 120 degrees. And this is independent of the line distance.

An output single wire is divided by number of loads. Each load receives a signal with its own parameters. For this purpose, set of converters have been proposed, so that $C 2-1$ and $C$ 3-1 in transmitter converts to $C 1-2$ and $C 1-3$ in receiver.

It is well known that the main part of power electrical system cost is wires. In [1] it is shown also that for one wire system can use one of the wires which are used in three phase system. A calculation shows that one wire system can be three time cheaper than three phase system. 


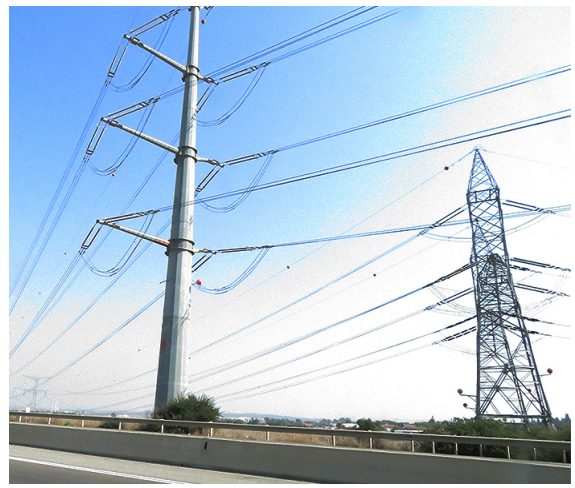

Figure 1. "Let my nature go".

\section{Fast Charging by One Wire}

Nowadays there is a problem associated with the need for fast charging of electrically driven vehicles. The time of charging depends on voltage in a charging device. The higher is the voltage, the shorter is the charging time. In residential areas the voltage is 380 volts, and with this voltage the time of charging is approximately 8 hours. This is generally acceptable in living areas or in employment areas.

However, there are situations when it is necessary to provide a fast charging, for example on highways or other places where a vehicle battery discharged. It is very expensive to erect high poles with high voltages wires along highways, and in many cases is just impossible. It is actually impossible to transmit a three-phase signal underground. It would be necessary to build underground tunnels, since a great distance must be provided between the wires.

For this problem solution was proposed charging system on basis of one wire method [6].

This system includes a converter for converting a three-phase electric current into an electric signal which can be transmitted through a single electric wire underground.

In this case one can use very height voltage.

The converting means include also a converter for converting the signal received from the single electric current transmission line into a three-phase electric current, one-phase current and DC.

So, using one wire method can be build fast charging stations in any places of long road.

\section{One Wire Method Can Be Used Not Only at a Low Frequency}

As a result, one of the most urgent tasks that does not yet have a solution can be completed: we mean a small-sized broadband antenna operating at all frequencies of its field. It is very well known that modern communication technology requires an antenna with such properties. Until now, small-sized broadband antennas work only at certain frequencies. 
This problem can be solved as follows: we take an unbalanced antenna. One end of the source goes to the emitter, the other to the nullifier. Due to a special shape of the emitter, current at each frequency "finds" a line with length of quarter of a wavelength. The size of the antenna depends on the lowest part. The upper frequency is not limited. At frequencies above six Gigahertz, the antenna has the highest gain in vertical direction. Thus, it can be used for communication with a satellite. These ideas and the results of simulations are described in detail in [7].

\section{Conclusions}

Dear students. As usual, every new technical proposal is met with doubts, disbelief and objections. If you embark on the path of engineering invention, you will hear:

- Nobodyneeds it,

- This contradicts the Ohm Law,

- Nobody wants to invest in this business,

- Are you smarter than everyone?

- Or like in Chekhov's story. "It is cannot be, because it is can never be." All the above was heard by the author plenty of times.

Nevertheless, it is worth looking for new ideas and solutions. It will make life more interesting and meaningful. The most interesting in life is to do something new.

\section{Conflicts of Interest}

The author declares no conflicts of interest regarding the publication of this paper.

\section{References}

[1] Bank, M. (2017) It Is Quite Another Electricity, Transmitting by One Wire and without Grounding (2nd Edition). Partridge Publishing, Singapore.

[2] Bank, M. (2016) Method and System for Reducing or Substantially Zeroing Electrical Potential. US Patent WO2017192448A1.

[3] Bank, M. (2014) Electrical Energy Transmission System with a Single Transmission Line. US Patent 9246405.

[4] Bank, M. (2018) Phase Converter for Vector Conversion of Three Phase Signals. US Patent 10305289 B1.

[5] Bronsthein, A. (2018) Universal Electrical Energy Transmission System. US Patent 9906032.

[6] Bank, M. (2018) System for Charging Electrically Drive Vehicles with a Single Line for Transmitting Electric Current from a Source to a Charging Station. US Patent 10250061. https://patents.justia.com/patent/10250061

[7] Bank, M. (2019) Wide Band Omni Directional Antenna. US Patent 10381744.

https://patents.justia.com/patent/10381744 